EPJ Web of Conferences 44, 04001 (2013)

DOI: $10.1051 /$ epjconf/20134404001

(C) Owned by the authors, published by EDP Sciences, 2013

\title{
Fluctuation theorem for an optically trapped tracer in dense colloids. A simulation study
}

\author{
Antonio M. Puertas ${ }^{1, \text { a }}$ \\ Group of Complex Fluids Physics, Department of Applied Physics, University of Almería, 04120 Almería, Andalucía, \\ Spain
}

\begin{abstract}
The work supplied by an external parabolic potential that traps one tracer in a colloidal system is studied in this work by computer simulations. The density of the bath is changed from zero up to values close to the glass transition, and the velocity varies over several decades from the linear behaviour in the low Peclet limit to the high Peclet limit. The work distributions are analyzed using the model for the isolated Brownian particle, where the friction coefficient and temperature of the medium have been fitted to reproduce the position distribution of the tracer in the trap. The overall agreement is good but not perfect. The region of negative works is studied in more detail using the predictions of the fluctuation theorem, finding good qualitative agreement with the model of the isolated Brownian particle. The present results indicate that the fluctuation theorem is of application in cases where the tracer dynamics is complex, as predicted by theoretical works.
\end{abstract}

\section{Introduction}

The fluctuation theorem (FT), developed in its various forms during the last two decades, has opened up and explained a new phenomenology of fundamental interest [1,2] with potential applications to diverse microscopic phenomena, particularly life [3]. In its general form, the FT states that the ratio of the probabilities of producing an entropy $\sigma_{\tau}$ and an entropy $-\sigma_{\tau}$ is positive and grows exponentially with $\sigma_{\tau}\left(\tau\right.$ indicates the time interval where $\sigma_{\tau}$ is measured). Noteworthy, the FT allows negative increments of entropy, as far as they are small, and the macroscopic Second Law of Thermodynamics is recovered for big entropies. The FT was first demonstrated in many particle systems with deterministic dynamics, but later also in stochastic systems [4], and with more complex dynamics [5]. The entropy is produced by dissipating the work done by an external force on (part of) the system by means of a viscous medium. This medium is characterized by a viscosity and its rheological behaviour sets, from a macroscopic point of view, how the energy is dissipated. Two important cases can be identified in the study of the FT: if the fluctuations are measured from an equilibrium ensemble, the transient form of the fluctuation theorem is obtained, which sets $P\left(\sigma_{\tau}\right) / P\left(-\sigma_{\tau}\right)=\exp \left(\beta \sigma_{\tau}\right)$; in contrast, if the fluctuations are measured in a (possibly far from equilibrium) stationary state, the previous result is valid only for large integration times, $\tau$.

In colloids, the FT has been derived in the overdamped limit, and experimentally tested in the transient regime for an isolated particle trapped in an external potential and dragged through the solvent $[6,7]$. On the other hand, optically trapped particles have been used experimentally to measure the local environment in dense quiescent systems, so called microrheology [8]. In passive microrheology the trap is held stationary [9-11], whereas in active microrhe-

a e-mail: apuertas@ual.es ology the tracer is dragged through the system [12-14]. The latter allows the measurement of microviscosities using the equality between average external and friction forces in the stationary regime, but its connection to the viscosity of the bulk system is still unclear $[15,16]$. The FT has been studied also in dense systems [17], in connection with plastic flow [18], or in other complex fluids such as a polymer melt [5]. In a system of hard spheres close to the glass transition, Williams and Evans found that time required for the steady state FT to apply increases with the density up to the glass point, whereas the transient from of the FT applies at all temperatures (in this case the tracer was pulled at constant force).

In this paper, we study the FT in dense colloidal systems using computer simulations, from diluted systems up to a volume fraction of $\phi=0.55$. A particle is trapped in a harmonic potential, that moves through the system at constant speed. The velocity varies several decades, from the linear behaviour at low velocity to the linear behaviour at large ones. In a previous publication, the distributions of tracer position inside the trap were analysed using the framework developed for an isolated Brownian particle: the maximum and width of the distribution are set by the friction coefficient and temperature, respectively; i.e., the bath is treated as a continuous medium. Upon increasing the trap speed, the effective friction coefficient shows a plateau for low velocities, then decreases and reaches a lower plateau at high speeds, whereas the effective temperature increases. Additionally, the dynamics of the tracer is affected by the high density of the bath and shows a position correlation function with a slowed decay, different from the single particle dynamics, and reflecting the slowing down of the bath collective dynamics due to the steric hindrance. In the present work we study the work distribution in the stationary state and check if the FT still applies for this system, despite the complex dynamics of the tracer. We find that using the e ffective parameters obtained from 
the position distributions reproduce satisfactorily the work distributions and its properties.

In the next section we give the details of the simulations and present the results of the tracer position distributions and correlation functions. In section 3 , the results are presented and discussed in connection with the previous results on this system and theoretical predictions, and the concluding remarks are given in section 4 .

\section{Simulation details}

In our simulations, the system is composed by 1000 quasihard particles undergoing damped Newtonian dynamics, to mimic colloidal dynamics. The equation of motion for particle $j$ is:

$$
m \ddot{\mathbf{r}_{j}}=\sum_{i} \mathbf{F}_{i j}-\gamma_{0} \dot{\mathbf{r}}_{j}+\mathbf{f}_{j}(t)\left(+\mathbf{F}_{\text {trap }}\right)
$$

where $\mathbf{F}_{i j}$ is the force of particle $i$ due to the soft-core potential $V\left(r_{i j}\right)=k_{B} T\left(r / \sigma_{i j}\right)^{-36}$, with $k_{B} T$ the thermal energy and $\sigma_{i j}$ the center to center distance, $\gamma_{0}$ is the friction coefficient of the solvent and $\mathbf{f}_{j}$ is a random force obeying the fluctuation dissipation theorem, $\left\langle\mathbf{f}_{i}(t) \mathbf{f}_{j}\left(t^{\prime}\right)\right\rangle=$ $6 k_{B} T \gamma_{0} \delta\left(t-t^{\prime}\right) \delta_{i j}$. The trap is felt only by one particle, and is introduced via a linear force: $\mathbf{F}_{\text {trap }}=-k\left(\mathbf{r}-\mathbf{r}_{\text {trap }}\right)$, where $\mathbf{r}_{\text {trap }}$ is the trap minimum. Polydispersity is included to avoid crystallization at high density - a flat distribution of radii $[0.9 a, 1.1 a]$ with $a$ the average particle radius. All particles have the same mass, $m$. In the units of the system, $a=1, k_{B} T=1$ and $m=1$, the friction coefficient is set to $\gamma_{0}=50$, and the spring constant $k=1000$.

In an equilibrated system, a particle is selected randomly and trapped in the external harmonic potential, with $\mathbf{r}_{\text {trap }}$ moving at constant velocity $v_{\text {trap }}$ along the $\mathrm{x}$-axis of the simulation box. To improve the statistics, the box is elongated along the dragging direction to dimensions $L_{x}, L, L$, with $L_{x}=8 L$. The particle is dragged for a distance $L_{x}$, whereas the rest of particles undergo Brownian motion (Eq. 1 without $\left.F_{\text {trap }}\right)$. Density is reported as volume fraction, $\phi=\frac{4}{3} \pi a^{3}\left[1+(\delta / a)^{2}\right] n_{c}$, with $n_{c}$ the particle concentration and $\delta$ the half-width of the radius distribution. The equations of motion were integrated using Heun's algorithm[19] with a time step $\delta t=5 \cdot 10^{-4}$. The position of the tracer with respect to the trap minimum is recorded for times up to $t=25000$, after a lag time $a / v_{\text {trap }}$, to assure stationarity. The use of damped Newtonian dynamics allows both momentum transfer to other particles and dissipation of energy to the fluid, in contrast to Newtonian dynamics or pure Brownian dynamics.

The analysis of the stationary tracer position distribution in the trap was performed previously [14] using the model for an isolated Brownian particle [20]. For this model, this distribution is given by

$$
p(\alpha)=\sqrt{\frac{k}{2 \pi k_{B} T}} \exp \left\{-k \alpha^{2} / 2 k_{B} T\right\}
$$

where $\alpha$ is $x-x_{s t}, y$ or $z$, with $x_{s t}$ the solution of the stationary state equation, i.e. $\gamma_{0} v_{\text {trap }}=-k\left(x_{s t}-x_{\text {trap }}\right)$. For finite density, $\gamma$ and $T$ must be fitted to reproduce the observed distribution, which indeed is Gaussian and is well modelled by the expression above with a suitable choice

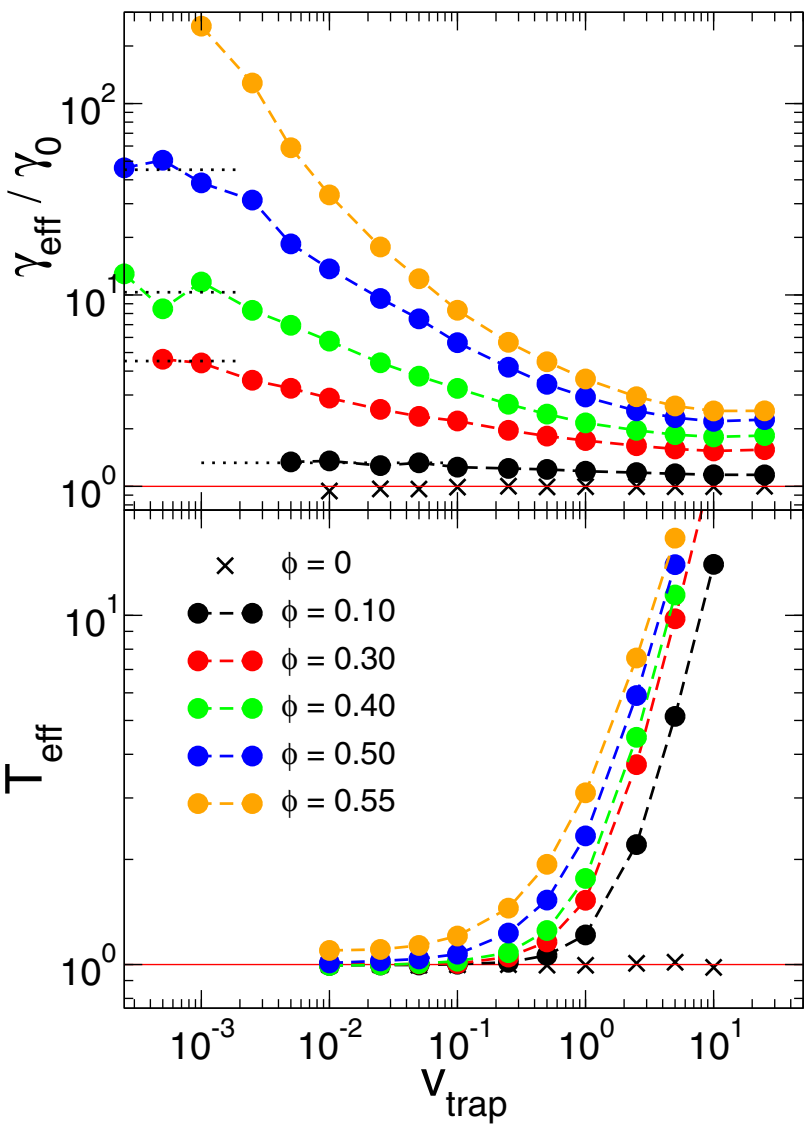

Fig. 1. Effective friction coefficient (upper panel) and effective temperature (lower panel) as a function of the trap velocity, as obtained from the analysis of the tracer position distributions, for different volume fractions, as labeled.

of these parameters [14]. The results of the fitting parameters, $\gamma_{e f f}$ and $T_{e f f}$, are presented in Fig. 1. The effective friction coefficient shows a high plateau at low velocities and a lower one at larger $v_{\text {trap }}$, whereas the effective temperature goes to the bath temperature in the low velocity limit and raises for larger ones. These results can be rationalized considering that the energy input is dissipated in the friction with the solvent, controlled by $\gamma_{0}$. For large velocities, the energy is injected in the system at a high rate, larger than the dissipation, resulting in a region around the tracer with increased mobility, i.e. larger local temperature.

This is confirmed by studying the tracer position correlation function, which measures how fluctuations of the tracer position relax to its equilibrium value. For an isolated Brownian particle, this correlation function reads for the $x$-coordinate:

$$
\langle\tilde{x}(t+\tau) \tilde{x}(t)\rangle=\frac{k_{B} T}{k} \exp \left(-k \tau / \gamma_{0}\right)+\left(v_{\text {trap }} \frac{\gamma_{0}}{k}\right)^{2} .
$$

where $\tilde{x}=x-x_{\text {trap }}$. This is presented in Fig. 2 for different densities at constant trap speed. Upon increasing the density, the correlation function shows a clear deviation from the single exponential decay, in connection with the different mechanisms involved in the tracer dynamics (friction with the solvent and collisions with other particles). Also, other features related to the behaviour of $\gamma_{e f f}$ and $T_{e f f}$, are observed in the figure: overall increase of the curves (due to the increasing temperature) and displacement of 


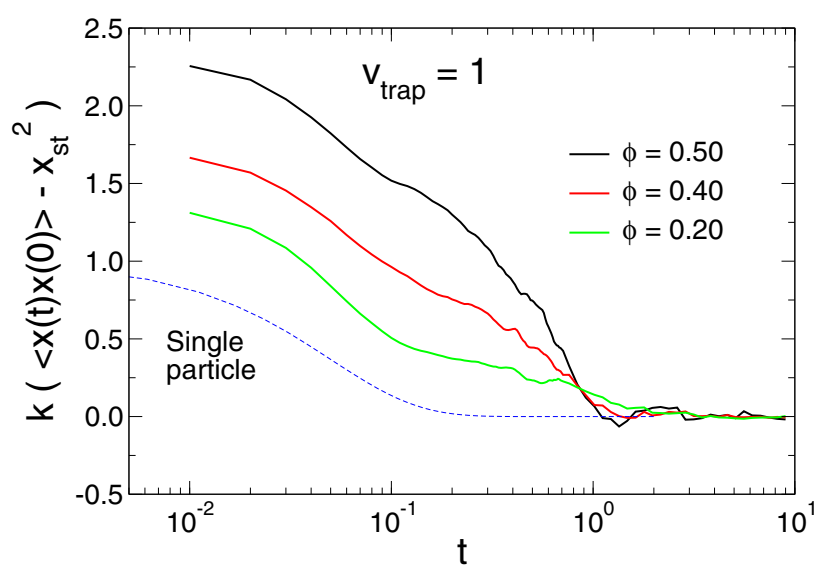

Fig. 2. Tracer position correlation functions for different densities, as labeled, at a constant trap velocity $v_{\text {trap }}=1$. Note that the long time plateau is substracted in all cases, to allow comparison of the different states.

the global decay to longer times (due to the increase of the friction coefficient).

\section{Results and discussion}

The work done on the particle by the trap during a time interval $\tau, W_{\tau}$, is then dissipated by the medium or transferred to other particles, and ultimately dissipated. For an isolated particle in a medium with friction and neglecting the inertial term, this work is Gaussian distributed, and in the stationary state reads $[21,22]$ :

$$
P_{S}\left(W_{\tau}\right)=\frac{\exp \left\{-\left[W_{\tau}-M_{S}(\tau)\right]^{2} / 2 V_{S}(\tau)\right\}}{\sqrt{2 \pi V_{S}(\tau)}}
$$

where $M_{S}(\tau)=\gamma_{0} v_{\text {trap }}^{2} \tau$ is the average work, and $V_{S}(\tau)=$ $2 \gamma_{0} k_{B} T v_{\text {trap }}^{2}\left(\tau-\tau_{r}\left[1-e^{-\tau / \tau_{r}}\right]\right)$, with $\tau_{r}=\gamma_{0} / k[21,22]$. In this model, this work is dissipated to the medium directly. This expression correctly reproduces the work distribution of the isolated particle in the simulations, as shown below.

In the simulations, $W_{\tau}$ is calculated as:

$$
W_{\tau}=\int_{0}^{\tau} d t \mathbf{v}_{\text {trap }} \mathbf{F}_{\text {trap }},
$$

The effect of the time interval is presented in Fig. 3, where the distributions for $\phi=0.40$ and $v_{\text {trap }}=0.1$ are presented for different values of $\tau$. Note that the average work is always positive, but the distributions extend to negative values of the work, indicating that the particle does work on the trap, i.e. the particle is pulling the trap. As expected, this tail disappears for large $\tau$, recovering the macroscopic form of the Second Law of Thermodynamics. All the distributions are Gaussian, as predicted by the single particle model, Eq. 4, and are qualitatively reproduced by the model if the solvent parameters (friction coefficient and temperature) are substituted by the effective parameters obtained from the analysis of the steady tracer position distributions.

The work distributions for different densities and velocities are presented in Fig. 4, and compared with the

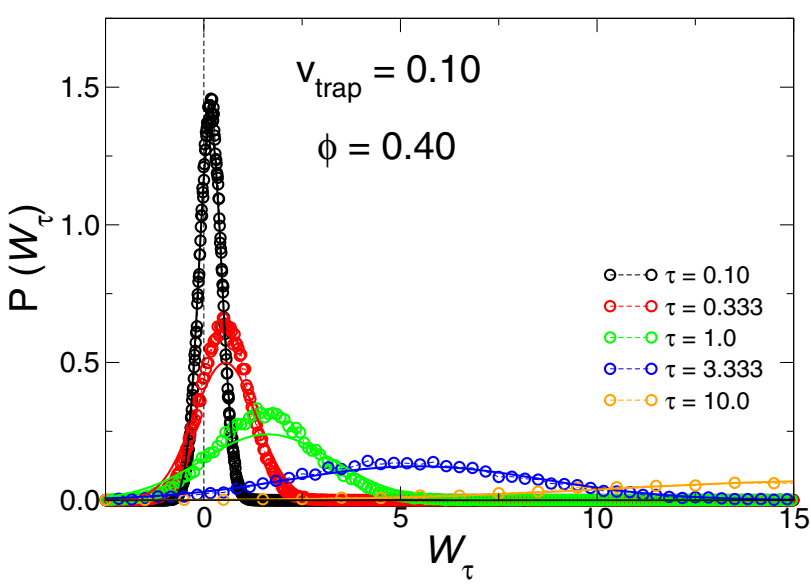

Fig. 3. Distribution of work done by the trap on the particle for $v_{\text {trap }}=0.10$ and $\phi=0.40$ and different time intervals, as labeled. The circles are data from simulations and the lines predictions from eq. 4.

theoretical calculations. As mentioned above, the distributions are Gaussian, except at low density and high velocity (see the red points in the lower panel). Upon increasing the density (at constant trap velocity), more work is dissipated to the medium, because its effective viscosity is higher, and the distribution is wider since the temperature is also higher. For faster velocities, much larger works are done by the trap, increasing as $v_{\text {trap }}^{2}$, although the friction coefficient decreases. In this limit, the work is only positive, approaching the macroscopic Second Law of Thermodynamics. The solid lines represent the calculated work distribution with expression 4 using the effective parameters from the position distributions. The overall agreement between the calculated distributions and simulation results is good but not perfect (note that all parameters in Eq. 4 are fixed), particularly the maximum is very well reproduced, except at small density and large velocities. It is important to note that although two mechanisms are possible to dissipate the work (namely, friction of the tracer and collisions with other particles), these merge giving a Gaussian distribution with a single maximum. Only in the case of large velocities and low density the two mechanisms can be resolved, showing a distribution that deviates clearly from the Gaussian behaviour (red points in the lower panel).

The FT can be further tested in the region of small and negative works by checking the following property in the stationary state:

$$
\frac{P_{S}\left(W_{\tau}\right)}{P_{S}\left(-W_{\tau}\right)}=\exp \left\{\frac{\beta W_{\tau}}{1-\epsilon(\tau)}\right\}
$$

where $\epsilon(\tau)=\tau_{r}\left[1-e^{-\tau / \tau_{r}}\right] / \tau[21,22]$. As the time interval $\tau$ diverges $\epsilon(\tau \rightarrow \infty) \rightarrow 0$, and the above expression tends to the more conventional form of the FT: $P_{S}\left(W_{\tau}\right) / P_{S}\left(-W_{\tau}\right) \rightarrow$ $e^{\beta W_{\tau}}(\tau \rightarrow \infty)$. The latter is valid for all $\tau$ in the transient regime and has been experimentally tested for a single colloidal particle trapped in a harmonic potential $[6,7]$. The FT in the stationary state is tested in Fig. 5 for the same state as in Fig. 3. As predicted, $\ln \left(P_{S}\left(W_{\tau}\right) / P_{S}\left(-W_{\tau}\right)\right)$ is indeed proportional to $W_{\tau}$, and the slope decreases with increasing integration times, and approaches 1 for large $\tau$.

In Fig. 6 the test of the FT is presented for different densities and two velocities, $v=0.10$ (upper panel) and 

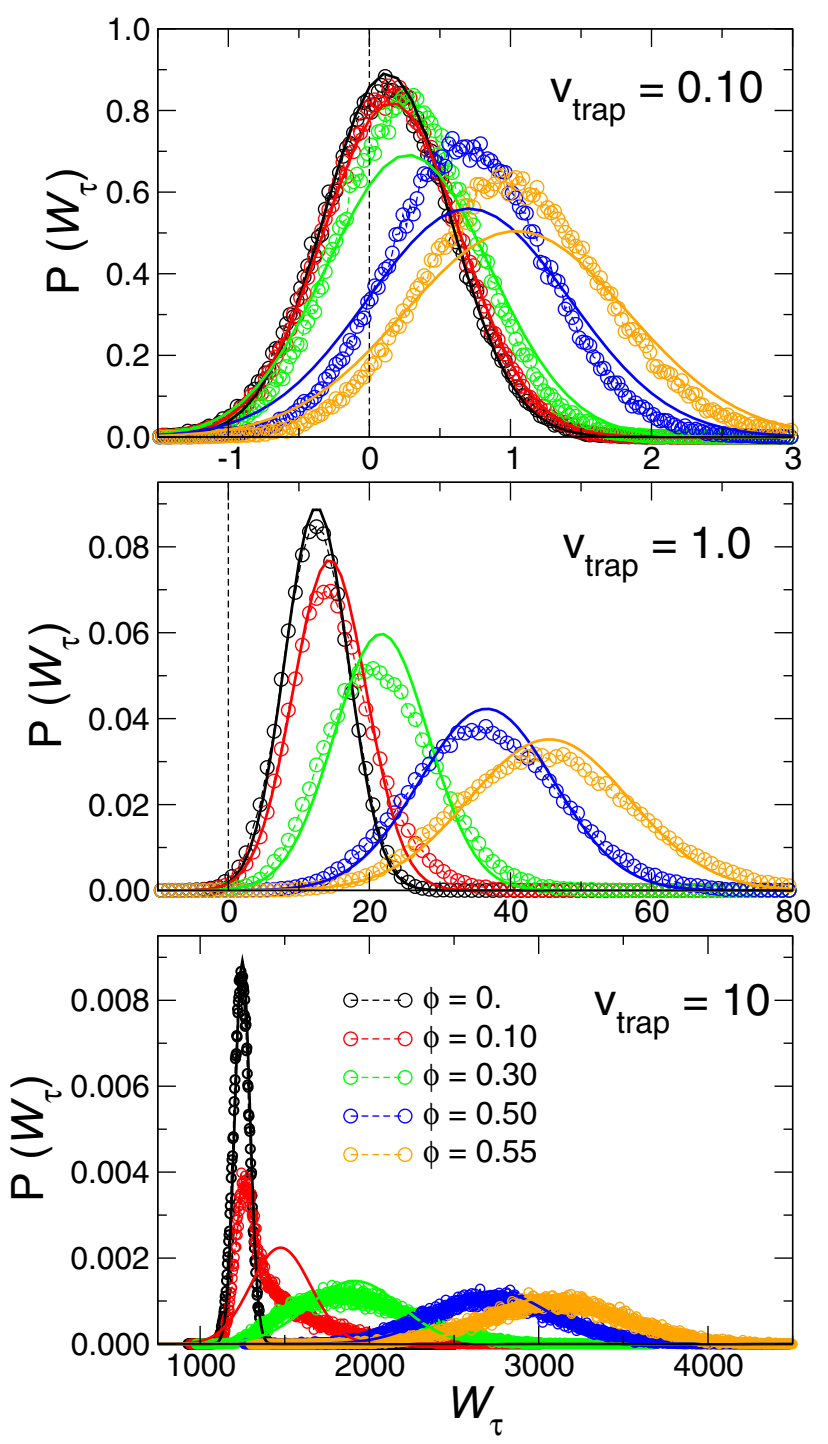

Fig. 4. Distribution of work done by the trap on the particle for $v_{\text {trap }}=0.10,1.0$ and 10 from top to bottom. Different densities are presented - note the di fferent-scale in the three panels. The circles are data from simulations and the lines predictions from eq. 4 with the effective parameters from the position distributions.

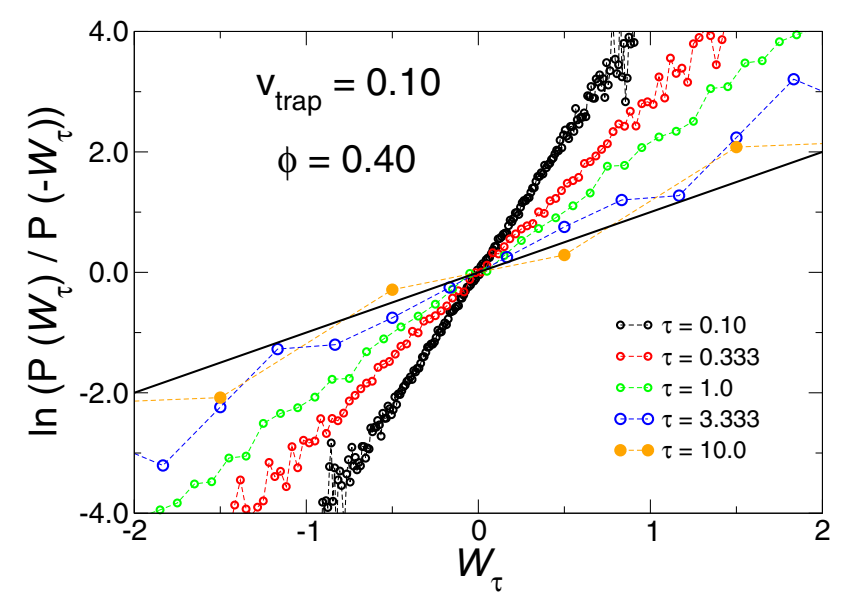

Fig. 5. Test of the FT for $v_{\text {trap }}=0.10$ and $\phi=0.40$ and different time intervals, as labeled. The thick black line represent the expected long time behaviour, slope 1 .
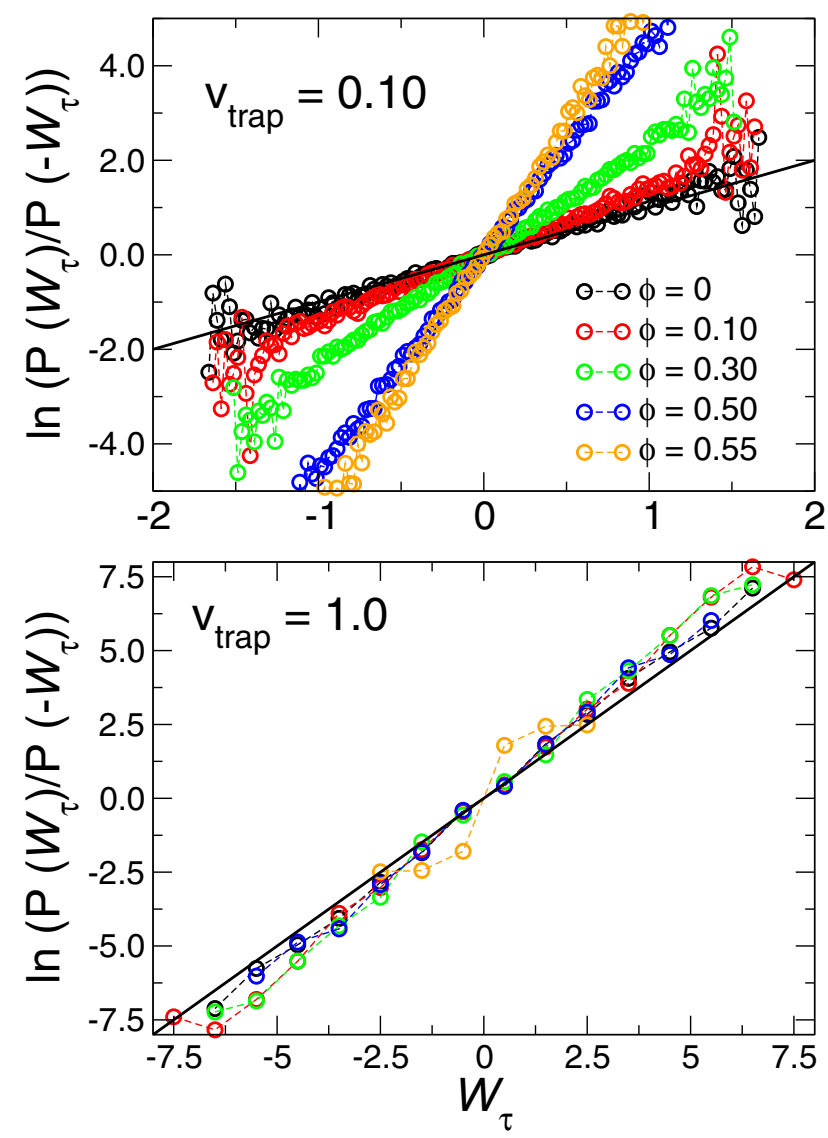

Fig. 6. Test of the FT for $v_{\text {trap }}=0.10$ (upper panel) and $v_{\text {trap }}=$ 1.0 (lower panel) and different volume fractions, as labeled. The thick black lines have slope 1 .

$v=1.0$ (lower panel). For the smaller velocity, the slope of the data increases with the volume fraction, starting from slope 1 for the dilute system, whereas for the larger velocity all the data almost collapse onto the same curve, with a slope close to 1 . These findings can be rationalized with the single-particle model as follows: because $\tau_{r}=\gamma / k$, upon increasing the volume fraction of particles, $\tau_{r}$ increases and so does $\epsilon(\tau)$ at constant $\tau$; the prefactor $1 /(1-\epsilon(\tau))$ thus increases in eq. 6, as observed in Fig. 6. For the larger velocity, the friction coefficient is smaller (see Fig. 1), and $\epsilon(\tau)$ is close to zero for this $\tau$.

These results are in agreement with other simulation studies [17] where a constant force is applied to the tracer instead of the parabolic trap used here. In both cases, upon approaching the glass transition, i.e. slowing down the system dynamics, $\ln \left(P_{S}\left(W_{\tau}\right) / P_{S}\left(-W_{\tau}\right)\right)$ deviates from $W_{\tau}$, confirming the expectations of the single particle approximation. On the theoretical side, Toyabe and Sano [5] corroborated that the FT can be formulated using the generalized Langevin equation, where the friction with the solvent contains a memory term, establishing a link with dense systems close to the glass transition.

\section{Conclusions}

The distribution of work supplied by an external parabolic potential moving at constant speed to a tracer in a colloidal system is studied using computer simulations and compared with the theoretical predictions for an isolated 
Brownian particle, i.e. treating the colloidal bath as continuous medium. The distribution of the tracer position in the trap was previously analyzed with the same model, and it was found that the friction coefficient and temperature of the bath needed to be fitted. Using these effective parameters, the work distributions have been calculated and compared with the simulation results, finding good agreement, particularly in the position of the maximum work. The agreement is worse in the case of the width of the distribution, but the overall trends are correctly captured. The fluctuation theorem is further tested by studying the ratio of positive to negative works, finding also that the expectations from the theory are fulfilled.

The present results give more evidence supporting that the fluctuation theorem can be applied to complex systems with non-simple dynamics with semi-quantitative agreement. In the present system, the particle dynamics shows a two-step decay due to the complex dynamics of the bath, approaching the glass transition. The dissipation of work is therefore performed via two routes: by direct friction with the solvent, and by collisions with other particles and their friction with the solvent, the latter being absent in the theoretical models used.

\section{References}

1. D.J. Evans, E.G.D. Cohen, G.P. Morriss, Phys. Rev. Lett. 712401 (1993).

2. D.J. Evans, D.J. Searles, Adv. Phys. 51, (2002) 15929.

3. C. Bustamante, J. Liphardt, F. Ritort, Phys. Today 58, (2005) 43.

4. J.L. Lebowitz, H. Spohn, J. Stat. Phys. 95, (1999) 333.

5. S. Toyabe, M. Sano, Phys. Rev. E 77, (2008) 041403.

6. G.M. Wang, E.M. Sevick, E. Mittag, D.J. Searles, D.J. Evans, Phys. Rev. Lett. 89, (2002) 050601.

7. D.M. Carberry et al. Phys. Rev. Lett. 92, (2004) 140601.

8. P. Cicuta, A.M. Donald, Soft Matter 3, (2007) 1449.

9. N. Greinert, T. Wood, P. Bartlett, Phys. Rev. Lett. 97, (2006) 265702.

10. B. Lukić, S. Jeney, Z. Sviben, A. J. Kulik, E.-L- Florin, and L. Forró, Phys. Rev. E, 76, 011112 (2007).

11. A.M. Puertas, AIP Proceedings 1319, (2010) 141.

12. A. Meyer, A. Marshall, B.G. Bush, E.M. Furst, J. Rheol. 50, (2006) 77.

13. I. Sriram, A. Meyer and E.M. Furst, Phys. Fluids 22, 062003 (2010).

14. L.G. Wilson, A.W. Harrison, W.C.K. Poon, A.M. Puertas. Europhys. Letters 93, (2011) 58007.

15. T.M. Squires, J.F. Brady, Phys. Fluids 17, (2005) 073101.

16. T.M. Squires, Langmuir 24, (2008) 1147.

17. S.R. Williams, D.J. Evans, Phys. Rev. Lett. 96, (2006) 015701.

18. J.A. Drocco, C.J. Olson Reichhardt, C. Reichhardt, Eur. Phys. J. E 34, (2011) 117.

19. W. Paul, D.Y. Yoon, Phys. Rev. E 52, (1995) 2076.

20. R.D. Astumian, J. Chem. Phys. 126, (2007) 111102.

21. O. Mazonka, C. Jarzynski, e-print cond-mat/9912121 (1999).

22. R. van Zon, E.G.D. Cohen, Phys. Rev. E 67, 046102 (2003). 